\title{
Offenheit als demokratisches Prinzip
}

\author{
Nikolaus Hamann
}

\section{Offenheit}

Offenheit - das ist ein Wort, das Erwartungen weckt und Möglichkeiten verspricht. Denken Sie nur an die "offene Gesellschaft", wie sie Karl Popper in den 50er Jahren gezeichnet hat, denken Sie an die "offene Zweierbeziehung" der sexuellen Revolution in der Folge von 1968, denken Sie an den Begriff "offenes Haus“, das Besuche ohne vorige Vereinbarung verspricht.

Auch heute sind wir mit einer Vielzahl von Begriffspaaren konfrontiert, die das Wort "offen"

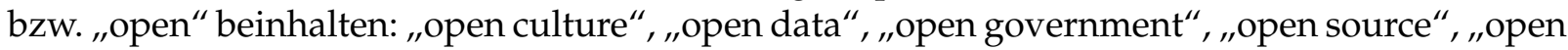
access", „open archiving" und viele viele mehr. Es gibt aber auch Auslegungen von „open", an die man nicht sofort denken würde. So versteht zum Beispiel der multinationale Konzern Procter \& Gamble „open innovation“ dahingehend, dass er die Weiterentwicklung bestehender bzw. die Erfindung neuer Produkte großteils auf die Konsument*innen auslagert und sich damit hohe Innovationskosten erspart.

Letztlich ist auch "Offenheit" oder „Openness" nur eine Worthülse, die mit Inhalten gefüllt werden muss. Welche Inhalte das sein können und werden, hängt auch davon ab, wer in der Gesellschaft seine Interessen besser durchsetzen können wird: Die großen Konzerne der Informationsbranche wie Google, Facebook etc., die mehr darauf setzen, dass wir offen (also gläsern) werden sowie die staatlichen wie privaten Informationssammler und Geheimdienste. Oder die Menschen in Wissenschaft und Kunst, die Studierenden, insgesamt die Bürgerinnen und Bürger aller Länder, also wir, deren Bedürfnis es ist, auf Wissen, Information und Werke der Kultur ohne Zeitverlust sowie ohne technische und finanzielle Beschränkungen zugreifen zu können. Es geht also auch hier um eine Machtfrage, und da kommen Politik und daher auch die Frage nach dem Wesen der Demokratie ins Spiel.

\section{Demokratie}

Das Wesen der Demokratie besteht darin, dass das Recht und die Macht vom Volk ausgehen. Wie dieses Volk oder - besser - die Gesellschaft strukturiert ist, fällt dabei nicht ins Gewicht. So wird zum Beispiel das alte Athen gerne als Wiege der Demokratie bezeichnet, obwohl wir alle wissen, dass nur ein kleiner Teil der Bevölkerung, nämlich die freien, wohlsituierten männlichen Bürger, stimmberechtigt war.

Die Länder des sowjetisch dominierten Systems bezeichneten sich in sinnloser Verdoppelung des Begriffs als Volksdemokratien, was formal richtig gewesen sein mag, aber durch das Diktat 
der kommunistischen Parteien konterkariert wurde. Ähnliche Kritik kann man zu Recht auch heute gegenüber sogenannten demokratischen Systemen im arabischen oder asiatischen Raum äußern.

Aber auch unsere westlichen repräsentativen Demokratien, mit ihrem System von Wahlen und Parlamenten, kommen dem Idealbild von Demokratie bestenfalls nahe, sind sie doch dadurch gekennzeichnet, dass die Bürger*innen ihre Vertretungen zwar frei wählen können, die wahre Macht aber doch in den Händen von nationalen und multinationalen Konzernen liegt. Die aktuellen - geheimen - Verhandlungen über die Handelsabkommen TTIP und CETA zeichnen ein klares Bild davon.

Die Entwicklung der digitalen Gesellschaften könnte allerdings eine Disruption der gängigen Auffassungen hinsichtlich Demokratie mit sich bringen. Würde die Digitalität doch unmittelbare, zeitnahe und weltumspannende Mitbestimmung der Menschen an allen sie betreffenden Fragen möglich machen. Die Einführung von World Wide Web und Internet mit allen damit verbundenen Möglichkeiten waren eine noch viel größere Disruption, als es die Erfindung des Buchdrucks mit beweglichen Lettern seinerzeit war.

Disruptionen in der gesellschaftlichen Wirklichkeit können also die Verhältnisse transformieren. Darauf müssen Politik und Recht reagieren. Wie darauf reagiert wird, ist wieder ein Ergebnis der gesellschaftlichen Machtverhältnisse, womit sich der Kreis zum Feld Demokratie schließt.

\section{Verknappung versus gemeinsames geistiges Eigentum}

"Privatisierung und Verknappung sind hinderlich und haben spätestens dann keine Berechtigung mehr, wenn es ums Überleben geht. Stattdessen sollten die Parolen gelten: Aufhebung aller Patente! Freiheit für den Erfindergeist! Kopiert global und schrankenlos! Macht Euch zu Diensten, was Euch nützlich ist! Schleift die Mautstellen! Entmachtet die Wegelagerer, die von Euch Zölle verlangen, wenn Ihr Euch bilden wollt!

Solche Appelle wären noch vor 20 Jahren ultraradikale Phrasen gewesen. Heute dagegen stehen die technischen Mittel bereit, um die Gesamtheit des [...] wichtigen Wissens so aufzubereiten, dass es möglichst viele verstehen, es so zu verbreiten, dass es alle Erdenmenschen unentgeltlich nutzen können, und so zu präsentieren, dass die Veränderung für eigene Zwecke möglich ist. Solche Darstellungen und Verbreitungen liegen im gemeinsamen Interesse der Menschheit. Deshalb sollten sie ausdrücklich mit dem Zweck des Kopierens und Adaptierens zur Verfügung stehen. Was man heute mit großem Aufwand zu verhindern versucht oder nur stillschweigend duldet, die Verbreitung ohne Zahlung, sollte zum expliziten Ziel werden. Was vom bürgerlichen Standpunkt wie ein gigantischer Raubzug aussieht, wäre tatsächlich eine Wallfahrt für das gemeinsame Wohl. ${ }^{\text {"1 }}$

Diese von Hans Thie, dem Wirtschaftsreferenten der Fraktion DIE LINKE im deutschen Bundestag, ursprünglich auf den „ökologischen Geist" gemünzten Sätze können unverändert auch für den "Geist der Offenheit" in Wissenschaft und Kunst verwendet werden, denn ",bei rein

\footnotetext{
${ }^{1}$ Hans Thie: Rotes Grün. Pioniere und Prinzipien einer ökologischen Gesellschaft. Eine Veröffentlichung der Rosa-Luxemburg-Stiftung,

http:/ / www.rosalux.de/fileadmin/rls_uploads/pdfs/sonst_publikationen/VSA_Thie_Rotes-Gruen.pdf, S. 99 f. (abgerufen 22.09.2017)
} 
geistiger Produktion, also dort, wo geistige Betätigung nicht nur Mittel, sondern Selbstzweck des Wirtschaftens ist, kommen die bürgerlichen Verhältnisse grundlegend ins Rutschen. Hier wanken die bisherigen Gesetze der Ökonomie und zugleich ihre bürgerlichen Formen. Hier ist tendenziell alles anders. Der Geist ist freier Commonist, nicht elitärer Bürger" ${ }^{2}$

In der Ökonomie des Geistes gilt laut Thie der Grundsatz, dass dieser umso produktiver sein kann, je allgemeiner und je mehr er der bürgerlichen Eigentumsform entrissen werden kann. Offenheit als politisches Prinzip bedeutet also, Ergebnisse der Wissenschaft und der Kunst nicht mehr primär in ihrer Warenform, sondern als weltweites gesellschaftliches Eigentum zu betrachten.

Dies bedeutet aber per se noch nicht, dass damit quasi automatisch die bürgerlichen Verhältnisse in eine neue, wie auch immer bezeichnete Gesellschaftsform transformiert werden würden, denn - wie die Initiativen vieler Verlage in Richtung Open Access zeigen - es lassen sich solche Modelle durchaus in die kapitalistische Verwertungslogik einbauen. Auch das intensive Engagement des US-Investors George Soros weist in diese Richtung. Auf jeden Fall aber hat das Prinzip Offenheit eine emanzipatorische Funktion und bedeutet eine Demokratisierung der gesellschaftlichen Verhältnisse, da sich Wissen und Werke der Kunst auf diese Art leichter und schneller verbreiten lassen, weniger leicht der Willkür der Verlage oder staatlicher Zensur unterworfen werden können, die weltweite Kommunikation fördern und daher auch den weniger entwickelten Ländern neue Chancen eröffnen. Bezieht sich das Prinzip Offenheit auch auf politische Entscheidungen und Akte der Verwaltung, so befördert dies die Transparenz derselben und gibt den Citoyens größere Chancen auf ihre Lebensumstände Einfluss zu nehmen.

\section{Open Access als Teilmenge des Prinzips Offenheit}

Geistige Produkte via Internet und WWW als öffentliches Gut zur Verfügung zu stellen ist der wesentliche Inhalt des Prinzips Offenheit in einer digitalisierten Welt. Diese Offenheit kann auf verschiedenen Ebenen und mit unterschiedlichen Wirkungen erreicht werden (Open Access, Open Data, Open Science, Open Source ... ). Für diesen Artikel soll vor allem Open Access beleuchtet werden, da dies für Bibliotheken die größte Relevanz besitzt.

„Die Idee des Open Access entspricht ganz den traditionellen Werten und Zielen akademischen Arbeitens, das auf Kollegialität, dem Austausch von Ideen und Ergebnissen, der gemeinsamen Suche nach Erkenntnis und der Verbreitung von Wissen zum Wohle der Gesellschaft insgesamt beruhen. Erst das digitale Zeitalter ermöglicht einen gemeinsamen freien Zugang zu wissenschaftlicher Erkenntnis und zu Forschungsdaten, wie er zuvor unter den Bedingungen des Druckzeitalters nicht denkbar war. Entscheidend dabei ist die Auswirkung dieses neuen Instruments auf die Entwicklung einer Gesellschaft, die den Leitbegriffen von Open Scholarship und Open Knowledge verpflichtet ist. ${ }^{\prime 3}$

\footnotetext{
${ }^{2}$ Hans Thie: Rotes Grün. Pioniere und Prinzipien einer ökologischen Gesellschaft. Eine Veröffentlichung der Rosa-Luxemburg-Stiftung, http:/ / www.rosalux.de/fileadmin/rls_uploads/pdfs/sonst_publikationen/VSA_Thie_Rotes-Gruen.pdf, S. 100 f. (abgerufen 22.09.2017)

${ }^{3}$ Hans-Jochen Schiwer: Es wird Zeit, alle alles lesen zu lassen, in: FAZ 05.06.2011, http:/ /www.faz.net/aktuell/berufchance/campus/open-access-es-wird-zeit-alle-alles-lesen-zu-lassen-1639084.html (abgerufen 22.09.2017)
} 
Ziel aller Open-Access-Initiativen ist es, Wissen und Information in digitaler Form für jedermann unentgeltlich und ohne technische oder rechtliche Barrieren dauerhaft zugänglich und nachnutzbar zu machen, was dem Fortschritt sowohl der Wissenschaft als auch der Menschheit insgesamt dient. Unterschiede gibt es in Bezug auf die Ausdehnung des Geltungsbereiches. Minimalforderung ist das Öffentlichmachen von Forschungsergebnissen, die mit Unterstützung der Öffentlichkeit erarbeitet wurden, die Maximalforderung bezieht auch Ergebnisse der Forschungsabteilungen der Wirtschaft bzw. solche, die in öffentlichen Institutionen (vor allem Universitäten und Fachhochschulen) im Auftrag der Wirtschaft erstellt wurden. In Hinblick auf die Maximalforderung werden sehr schnell die Grenzen der gegenwärtigen Open-Access-Politik sichtbar, denn Forschungsdaten zum Beispiel der Pharmaindustrie werden von den Wenigsten gedanklich - geschweige denn tatsächlich - dem Prinzip Offenheit unterworfen.

Exkurs:

Open Access (wie auch alle anderen Kategorien von Offenheit) kann nicht in gleicher Weise für Wissenschaft und Kunst gelten. Während Wissenschaftler*innen in der Regel ihre Arbeit in (mehr oder weniger) gesicherter Position vollbringen, sind freischaffende Künstler*innen sehr wohl darauf angewiesen, ihre Werke vermarkten zu können. Daher wird es - dies nur als Nebenbemerkung - notwendig sein, das Urheber*innenrecht nach Sparten getrennt zu reformieren oder die Einkommensfrage der Künstler*innen generell, etwa durch ein bedingungsloses Grundeinkommen, auf eine neue Basis zu stellen.

\section{Open Access und Nord-Süd-Beziehungen}

„The close link between scientific development and the social and economic wellbeing of a nation has long been recognised. Jawaharlal Nehru, India's first prime minister, said: ,It is science alone that can solve the problems of hunger and poverty, of insanitation and illiteracy... Who indeed can ignore science today? We need it at every turn.' Likewise, a 1982 UNESCO report states that assimilation of scientific and technological information is an essential precondition for progress in developing countries'. The InterAcademy Council says: ,In a world moving rapidly toward the knowledge-based economies of the 21st century, capacity building in science and technology (S\&T) is necessary everywhere. But the need is greatest for the developing countries. ${ }^{\prime \prime 4}$

Wie bereits im vorigen Abschnitt erwähnt, spielt Offenheit und damit auch Open Access in der Diskussion um gerechte Nord-Süd-Beziehungen eine große Rolle, oft aber in dem Sinn, dass der Norden in (post-)imperialistischer Großzügigkeit die ärmeren Länder an seinem Wissen teilhaben lassen will. Die sich entwickelnden Länder mögen diese Sichtweise naturgemäß nicht sehr gerne und stellen ihre ganz andere Argumente entgegen, warum die Zeit für Open Access bzw. Open Archiving reif sei.

Natürlich ist es wahr, dass die Länder des Südens auf Grund ihrer Armut noch viel weniger in der Lage sind als wir im globalen Norden, die exorbitanten Kosten für traditionell oder digital gespeichertes Wissen aufzubringen. Auch die Forschungsetats sind verständlicherweise

\footnotetext{
${ }^{4}$ Leslie Chan, Barbara Kirsop, Subbiah Arunachalam: Open access archiving: the fast track to building research capacity in developing countries. In: SciDevNet (11.02.2005),

http:/ / www.scidev.net/global/communication/feature/open-access-archiving-the-fast-track-to-building-r.html (abgerufen 22.09.2017)
} 
viel spärlicher bedacht. Aber die Länder des Südens haben auch eine Menge Wissen anzubieten, das bisher viel zu wenig beachtet wurde, vor allem im Gesundheits- oder Agrarsektor und Klimafragen. Das Ermöglichen eines gerechten Austauschs über Open Access würde also auch dem Norden großen Nutzen bringen.

Die Länder des Südens haben oft - auch das eine Folge von Imperialismus und Kolonialismus sehr konservative Strukturen für ihre Bildungs- und Forschungslandschaft übernommen. Dennoch haben diese Länder oft einen prozentuell wesentlich höheren Ausstoß an Open-AccessJournalen als manche Länder des Nordens. Das heißt auch strukturell kann gegenseitiges Lernen durchaus erfolgversprechend und lohnend sein.

\section{Grenzen und Kritik von Open Access}

Kritik an Open-Access-Strategien kam hauptsächlich aus den Reihen der Verleger und aus der Wirtschaft. Erstere war eindeutig vom möglichen Wegfall gewinnträchtiger Geschäftsfelder geleitet und somit von einer gesellschaftspolitischen Position widerlegbar, wird aber von manchen Wissenschaftlerinnen entweder aus einer traditionellen Denkweise heraus oder mit dem Argument unterstützt, Open-Access-Publikationen brächten nicht die gleiche Reputation wie Veröffentlichungen in herkömmlichen Journalen mit hohem Impact-Faktor und behinderten dadurch die wissenschaftliche Karriere.

Die Unternehmen sind oftmals Auftraggeberin für angewandte Forschung und fürchten aus Konkurrenz- und Profitgründen natürlich, durch Open Access Ergebnisse der von ihnen beauftragten Forschung Mitbewerbern gegenüber nicht mehr geheim halten zu können. Gleichzeitig profitiert die Wirtschaft aber auch von schnellerem und kostenfreiem Zugang zu Information. Hier ist genau der Punkt erreicht, wo die Diskussion von der Ebene der allgemeinen Sinnhaftigkeit zur Ebene der Gesellschaftsordnung führt, wo also die Debatte zwischen Weiterentwicklung der bürgerlichen Demokratie oder einem anderen Gesellschaftsmodell geführt werden muss.

Kritik an der Verortung von Open Access innerhalb der bürgerlichen Ordnung kommt aus linken Gruppen. Moniert wird einerseits die ideologische Begründung für Open Access im imperialistischen Diskurs einer "Entwicklungshilfe“ - dazu siehe oben - aber auch die Tatsache, dass Open Access als technische Lösung allein bestehende Ungleichheiten nicht zu vermindern vermag. „[Es] zeigt die aktuelle Definition der Golden Road des Open Access, dass es gerade die großen Wissenschaftsverlage sind, die es sehr genau verstanden haben, Open Access für sich und damit für eine weitergehende Vormachtstellung zu nutzen. War mit der Golden Road ursprünglich nur gemeint, Wissenschaftliche Beiträge offen und frei erstzuveröffentlichen (im Gegensatz zur Green Road, der freien und offenen Zweitveröffentlichung nach einer exklusiven Vermarktung der Beiträge durch privatwirtschaftliche Verlage), wird ,golden' mittlerweile mehrheitlich so verstanden, dass privatwirtschaftliche Verlage nach Zahlung einer Publikationsgebühr die offene und freie Erstveröffentlichung der wissenschaftlichen Beiträge organisieren. ${ }^{\prime 5}$ Eingefordert wird eine „faire[n] Teilhabe der Fabrikbelegschaften, Konsument*innen oder anderen Ideengeber*innen an den Ergebnissen. ${ }^{\prime 6}$

\footnotetext{
5 Joerg Braun: Ohne Gleichberechtigung und sozialen Ausgleich bleibt Open dicht. In: Digitale Linke (10.07.2012), http:/ / blog.die-linke.de/digitalelinke/ohne-gleichberechtigung-und-sozialen-ausgleich-bleibt-open-dicht / (abgerufen 22.09.2017)

${ }^{6}$ Joerg Braun: Ohne Gleichberechtigung und sozialen Ausgleich bleibt Open dicht. In: Digitale Linke (10.07.2012),
} 


\section{„Geistiges Eigentum“}

"The debate on the value of open access to publicly funded research information is now migrating from 'whether' to 'how'. ${ }^{\prime 7}$ Die Open-Access-Bewegung hat eine Breite erreicht, die eine Implementierung auf jeden Fall erforderlich macht, unabhängig davon, ob damit eine Reform des Publikationsverhaltens innerhalb der bürgerlichen Ordnung gemeint ist oder darüber hinaus dieser Prozess als Teilbereich einer Transformation der gesellschaftlichen Verhältnisse dienen soll.

Hinter der Debatte um Open Access steht die grundsätzliche Frage, wem Wissen eigentlich gehört, und das führt schnurgerade zur Diskussion um "geistiges Eigentum", die eine zutiefst ethische und daher natürlich auch politische ist.

Es gibt eine ganze Reihe von Gründen, die darauf hinweisen, dass Institutionen zum Schutz sogenannten "geistigen Eigentums"

- das Wirtschaftswachstum insgesamt eher hemmen und daher nur ein suboptimales Wohlstandsniveau ermöglichen, da durch den Schutz von sogenanntem "geistigen Eigentum" nur eine suboptimale Allokation von Wissen zur Steigerung von Effektivität und Effizienz im Umgang mit knappen Gütern möglich ist;

- zur Reproduktion oder gar Verstärkung sozialer Ungleichheit beitragen;

- aufgrund der technischen Voraussetzungen, die zu ihrer Durchsetzung notwendig sind, antiliberale bzw. totalitäre Entwicklungen begünstigen. ${ }^{\prime 8}$

Insgesamt lässt sich aus der Entwicklung der Schutzrechte für "geistiges Eigentum“ ablesen, dass es eine stetige Tendenz hin zu den Rechten von Verwertungsorganisationen gibt, wohingegen die Rechte der Urheber*innen, der Nutzer*innen und insgesamt der Öffentlichkeit immer weniger Beachtung finden. Das im 18. Jahrhundert als gerechter Ausgleich zwischen den verschiedenen Interessen von Urheber*innen, Verwertern, Nutzer*innen und Öffentlichkeit angelegte Kräfteparallelogramm ist aus dem Gleichgewicht geraten. Gerechtigkeit ist aber eines der Grundthemen der Ethik, genauso wie die Ermöglichung eines "guten Lebens" für alle, was hier sowohl philosophisch als auch sozial-ökonomisch gemeint ist. Dabei ist aber - wie bereits erwähnt - zu beachten, dass Open-Access-Strategien nicht eins zu eins auf künstlerische Produktion umgelegt werden können. Hier muss vielmehr das Urheber*innenrecht („,das Arbeitsrecht der Kreativen“9 ) so geändert werden, dass Künstler*innen erstmals (!) in ihrer Gesamtheit die Chance auf ein "gutes Leben“ bekommen.

http://blog.die-linke.de/digitalelinke/ohne-gleichberechtigung-und-sozialen-ausgleich-bleibt-open-dicht/ (abgerufen 22.09.2017)

${ }^{7}$ Leslie Chan, Barbara Kirsop, Subbiah Arunachalam: Open access archiving: the fast track to building research capacity in developing countries. In: SciDevNet (11.02.2005),

http:/ / www.scidev.net/global/communication/feature/open-access-archiving-the-fast-track-to-building-r.html (abgerufen 22.09.2017)

${ }^{8}$ Eissler, Stephan: Eine Kritik der Institution des so genannten „geistigen Eigentums“ im digitalen Zeitalter aus Perspektive liberaler Theorien. Vortrag auf der 3. Oekonux-Konferenz in Wien im Mai 2004,

http:/ / dritte.oekonux-konferenz.de/dokumentation/texte/Eissler.pdf , (abgerufen am 30.09.2017), S. 46

${ }^{9}$ Daniel Leisegang: Die Zukunft des Wissens Google Books, Open Access und die Informationsgesellschaft von morgen. In: Blätter für deutsche und internationale Politik 53 (11) 2009,

http:/ / www.blaetter.de/archiv/jahrgaenge/2009/november/die-zukunft-des-wissens (abgerufen 22.09.2017) 
N. Hamann

LIBREAS. Library Ideas, 32 (2017).

Nikolaus Hamann. Bibliothekar i.R., Vorstandsmitglied der Vereinigung österreichischer Bibliothekarinnen und Bibliothekare (VÖB), Koordinator des Arbeitskreises kritischer Bibliothekarinnen und Bibliothekare (KRIBIBI), http:/ / www.kribibi.at 